Proceedings of the Edinburgh Mathematical Society (2003) 46, 435-450 (C)

DOI:10.1017/S0013091502000846 Printed in the United Kingdom

\title{
PROJECTIVE DESCRIPTION OF WEIGHTED (LF)-SPACES OF HOLOMORPHIC FUNCTIONS ON THE DISC
}

\author{
KLAUS D. BIERSTEDT ${ }^{1}$ AND JOSÉ BONET ${ }^{2}$ \\ ${ }^{1}$ Institut für Mathematik, Universität Paderborn, D-33095 Paderborn, \\ Germany (klausd@uni-paderborn.de) \\ ${ }^{2}$ Departamento de Matemática Aplicada, ETS Arquitectura, \\ Universidad Politécnica de Valencia, E-46071 Valencia, \\ Spain (jbonet@mat.upv.es)
}

(Received 5 September 2002)

Dedicated to Hans Jarchow and Dietmar Vogt on the occasion of their 60th birthdays

\begin{abstract}
The topology of certain weighted inductive limits of Fréchet spaces of holomorphic functions on the unit disc can be described by means of weighted sup-seminorms in case the weights are radial and satisfy certain natural assumptions due to Lusky; in the sense of Shields and Williams the weights have to be normal. It turns out that no assumption on the (double) sequence of normal weights is necessary for the topological projective description in the case of o-growth conditions. For O-growth conditions, we give a necessary and sufficient condition (in terms of associated weights) for projective description in the case of (LB)-spaces and normal weights. This last result is related to a theorem of Mattila, Saksman and Taskinen.
\end{abstract}

Keywords: normal weight; associated weight; topological subspace

2000 Mathematics subject classification: Primary 46E10

Secondary 30H05; 46A13; 46M40

\section{Introduction}

Weighted inductive limits of spaces of holomorphic functions play an important role in fields like partial differential equations and convolution equations, distribution theory, spectral theory and complex analysis. We deal with the case in which the topologies of the step spaces of the inductive limit are given by weighted sup-seminorms. In general, however, the locally convex inductive limit topology is very intricate. For applications and direct calculations, it is useful to know when projective description holds, i.e. when the inductive limit topology is also given by a (natural) system of weighted sup-seminorms.

The first general projective description results were given by Bierstedt, Meise and Summers in [8] (see also [6]). It turned out that, in a very general framework, in the case of o-growth conditions the weighted inductive limit $\mathcal{V}_{0} C(X)$ of spaces of continuous functions is always a topological subspace of its projective hull $C \bar{V}_{0}(X)$. The proof of the 
corresponding result [8, Lemmas 1.1 and 1.2 and Theorem 1.3] worked with partitions of unity and showed that the topologies of both spaces coincide on the dense subspace of functions with compact support. The main projective description theorem for (LB)spaces $\mathcal{V} C(X)$ of continuous functions with O-growth conditions involved condition (D) on the sequence $\mathcal{V}=\left(v_{n}\right)_{n}$ of weights $(\mathrm{cf}$. [7]). Projective description for weighted (LF)spaces of continuous functions was treated in our article [4]; here the conditions (wQ) and $(\mathrm{Q})$, due to Vogt, are relevant.

Most of the techniques of proof used in the case of weighted inductive limits of spaces of continuous functions do not work for spaces of holomorphic functions. In the case of (semi-) Montel (LB)-spaces of holomorphic functions, the Baernstein Lemma applies and helps to deduce projective description for weighted inductive limits of spaces of holomorphic functions from results for the corresponding spaces of continuous functions. But, unfortunately, for proper (LF)-spaces no analogue of the Baernstein Lemma holds, and the situation is much more complicated in this case.

Counterexamples for projective description in (LB)-spaces of holomorphic functions were given only relatively recently, by Bonet and Taskinen [13], Bonet and Melikhov [13] and Bonet and Vogt [14]. The recent paper [11] of Bonet and Meise gives new insight into counterexamples for (LF)-spaces of holomorphic functions. For a survey of the state of the art in projective description for spaces of holomorphic functions see [3].

While most projective description results invoke only conditions on the sequence of weights, but no additional conditions on the individual weights, Mattila, Saksman and Taskinen $[\mathbf{2 2}]$ proved projective description for $(\mathrm{LB})$-spaces $\mathcal{V} H(\mathbb{D})$ of holomorphic functions on the unit disc $\mathbb{D}$ with O-growth conditions under condition (D) if all the weights belong to a certain class. Such weights had been called normal by Shields and Williams [26], but it is the form of the conditions (L1), (L2) of Lusky [20, 21] which we will use here. In fact, it is clear that the present paper owes much to the articles of Lusky and Mattila, Saksman and Taskinen: we work in a similar setting and use certain of their techniques, but in a somewhat different way.

Our main result on (LF)-spaces of holomorphic functions with o-growth conditions (Theorem 3.1) asserts that, whenever all the weights $v_{n, k}$ in the (double) sequence $\mathcal{V}$ are normal in the sense of $[\mathbf{2 6}]$, then the weighted inductive limit $\mathcal{V}_{0} H(\mathbb{D})$ is always a topological subspace of its projective hull $H \bar{V}_{0}(\mathbb{D})$. This is somehow parallel to the aforementioned result of $[\mathbf{8}]$ for spaces of continuous functions, which, in fact, is also used in our proof. We again show that the topologies coincide on a dense subspace; this time it is the subspace of all polynomials. It follows (Corollary 3.2) from a result of $[4]$ that $\mathcal{V}_{0} H(\mathbb{D})=H \bar{V}_{0}(\mathbb{D})$ holds algebraically and topologically if the sequence of normal weights satisfies condition $(\mathrm{Q})$. There is a natural biduality between $\mathcal{V}_{0} H(\mathbb{D})$ and $\mathcal{V} H(\mathbb{D})$ (see $[\mathbf{5}])$, but, unfortunately, this allows us to prove projective description for $\mathcal{V} H(\mathbb{D})$ under condition (wQ) (Corollary 3.3) only if it is known that the inductive limit topology has a system of 0-neighbourhoods which are closed in the compact open topology.

On the other hand, in the case of (LB)-spaces of holomorphic functions with O-growth conditions, a slight modification of the proof of Theorem 3.1 allows us to prove (Theo- 
rem 4.1) that if $\mathcal{V}=\left(v_{n}\right)_{n}$ is a decreasing sequence of normal weights on $\mathbb{D}$, then $\mathcal{V} H(\mathbb{D})$ is always a topological subspace of the corresponding inductive limit $\mathcal{V} C(\mathbb{D})$ of spaces of continuous functions. From the main result of [7], one then obtains (Corollary 4.2) that $\mathcal{V} H(\mathbb{D})=H \bar{V}(\mathbb{D})$ algebraically and topologically whenever the sequence $\mathcal{V}=\left(v_{n}\right)_{n}$ of normal weights satisfies condition (D), recovering the projective description result of $[\mathbf{2 2}]$. In terms of the associated weights (cf. [10]), it is even possible to give a necessary and sufficient condition (Theorem 4.4) for the topological projective description $\mathcal{V} H(\mathbb{D})=H \bar{V}(\mathbb{D})$ in the case of normal weights on $\mathbb{D}$.

The paper is organized as follows. We collect definitions and preliminaries in $\S 2$. In $\S 2.1$, a class $\mathcal{W}$ of weights is introduced; for the weights in this class and a sequence $\left(R_{n}\right)_{n}$ of operators from the space $H(\mathbb{D})$ of all holomorphic functions on $\mathbb{D}$ into itself, certain conditions (P1) and (P2) are considered. Later on, in the final section, $\S 5$, it is shown that the conditions (L1) and (L2) on the weights, recalled in $\S 2.1$, imply the conditions (P1) and (P2), where $R_{n}$ is the arithmetic mean of the partial sums of the Taylor series of order $2^{n}, \ldots, 2^{n+1}-1$. In $\S 2.2$, weighted (LF)-spaces of holomorphic functions on the disc and their projective hulls are introduced, and projective description is discussed in more detail. Our main results and their proofs are collected in $\S \S 3$ and 4 . Section 3 is devoted to the results on (LF)-spaces of holomorphic functions with o-growth conditions; $\S 4$ deals with (LB)-spaces of holomorphic functions with O-growth conditions.

\section{Notation, preliminaries}

\subsection{Class $\mathcal{W}$, operators $\boldsymbol{R}_{n}$, conditions (P1), (P2), (L1), (L2)}

Let $\mathcal{W}$ be a class of strictly positive continuous radial weights $v$ on the unit disc $\mathbb{D}$ which satisfy $\lim _{r \rightarrow 1-} v(r)=0$ and for which the restriction of $v$ to $[0,1)$ is non-increasing. We suppose that the class $\mathcal{W}$ is stable under finite minima and under multiplication by positive scalars.

For each $v \in \mathcal{W}$ we define the following weighted Banach spaces of holomorphic functions on $\mathbb{D}$ :

$$
\begin{aligned}
H v(\mathbb{D}) & :=\left\{f \in H(\mathbb{D}) ;\|f\|_{v}:=\sup _{z \in \mathbb{D}} v(z)|f(z)|<\infty\right\}, \\
H v_{0}(\mathbb{D}) & :=\{f \in H(\mathbb{D}) ; v|f| \text { vanishes at } \infty \text { on } \mathbb{D}\} .
\end{aligned}
$$

Next, we assume that there is a sequence $R_{n}: H(\mathbb{D}) \rightarrow H(\mathbb{D}), n \in \mathbb{N}$, of linear operators which are continuous for the compact open topology (co) and such that the range of each $R_{n}$ is a finite-dimensional subspace of the polynomials. It is also assumed that $R_{n} R_{m}=R_{\min (n, m)}$ holds for each $n, m$ with $n \neq m$ and that for each polynomial $p$ there is $n$ such that $R_{n} p=p$, from which it follows that $R_{m} p=p$ for each $m \geqslant n$. Moreover, we suppose that there is $c>0$ such that $\sup _{|z|=r}\left|R_{n} p(z)\right| \leqslant c \sup _{|z|=r}|p(z)|$ for each $n$, each $r \in(0,1)$ and each polynomial $p$.

Finally, setting $R_{0}:=0$, and putting $r_{n}:=1-2^{-n}, n \in \mathbb{N} \cup\{0\}$, we assume that the following conditions are satisfied by the class $\mathcal{W}$. 
(P1) There is $C \geqslant 1$ such that for each $v \in \mathcal{W}$ and for each polynomial $p$,

$$
\begin{aligned}
\frac{1}{C} \sup _{n}\left(\sup _{|z|=r_{n}}\left|\left(R_{n+2}-R_{n-1}\right) p(z)\right|\right) v\left(r_{n}\right) & \leqslant\|p\|_{v} \\
& \leqslant C \sup _{n}\left(\sup _{|z|=r_{n}}\left|\left(R_{n+1}-R_{n}\right) p(z)\right|\right) v\left(r_{n}\right) .
\end{aligned}
$$

(P2) For each $v \in \mathcal{W}$ there is $D(v) \geqslant 1$ such that for each sequence $\left(p_{n}\right)_{n}$ of polynomials of which only finitely many are non-zero,

$$
\sup _{z \in \mathbb{D}}\left|\sum_{n=1}^{\infty}\left(R_{n+1}-R_{n}\right) p_{n}(z)\right| v(z) \leqslant D(v) \sup _{k}\left(\sup _{|z|=r_{k}}\left|p_{k}(z)\right|\right) v\left(r_{k}\right) .
$$

The main example for $\mathcal{W}$ will be the set of all the strictly positive continuous radial weights $v$ on $\mathbb{D}$ which satisfy $\lim _{r \rightarrow 1-} v(r)=0$, which are non-increasing on $[0,1)$, and such that there are $\varepsilon_{0}>0$ and $k(0) \in \mathbb{N}$ with the following conditions:

$$
\begin{aligned}
& \text { (L1) } \quad \inf _{k} \frac{v\left(r_{k+1}\right)}{v\left(r_{k}\right)} \geqslant \varepsilon_{0}, \\
& \text { (L2) } \quad \limsup _{k \rightarrow \infty} \frac{v\left(r_{k+k(0)}\right)}{v\left(r_{k}\right)}<1-\varepsilon_{0} .
\end{aligned}
$$

In this case, $R_{n}$ is the convolution with the de la Vallée Poussin kernel. The conditions (L1) and (L2) form a uniform version of the conditions introduced by Lusky in [20, 21], and they also appear in the sequence space representations for weighted (LB)-spaces given by Mattila, Saksman and Taskinen $[\mathbf{2 2}]$. We will show in the last section that the conditions (L1), (L2) imply the conditions (P1), (P2).

A function $f$ defined on an interval of the real line is called almost decreasing (respectively, almost increasing) if there is $C>0$ such that $x<y$ implies $f(y) \leqslant C f(x)$ (respectively, $f(x) \leqslant C f(y)$ ). By $[\mathbf{1 5}$, Lemma 1.(a)], a radial weight $v$ on $\mathbb{D}$ satisfies (L1) for a certain $\varepsilon_{0}$ if and only if it satisfies the following condition (U) of Shields and Williams [26, p. 5].

There is $q>0$ such that for the function $\psi, \psi(t):=1 / v(1-(1 / t))$ for $t \geqslant 1$, $t \rightarrow \psi(t) / t^{q}$ is almost decreasing.

In fact, $\varepsilon_{0}$ and $q$ depend on each other. On the other hand, [15, Lemma 1.(b)] implies that a radial weight $v$ on $\mathbb{D}$ satisfies (L2) for a certain $\varepsilon_{0}$ and a certain $k(0)$ if and only if $v$ satisfies the following condition (L) of Shields and Williams [26, p. 5].

There is $p>0$ such that, for the function $\psi$ defined above, $t \rightarrow \psi(t) / t^{p}$ is almost increasing.

Again, $\varepsilon_{0}$ and $k(0)$ depend on $p$ and vice versa. Shields and Williams called weights satisfying both (L) and (U) normal weights. 


\subsection{Weighted (LF)-spaces of holomorphic functions, projective description}

We now recall the definition of the weighted (LF)-spaces of holomorphic functions on $\mathbb{D}$. Let $\mathcal{V}=\left(v_{n, k}\right)_{n, k \in \mathbb{N}}$ be a (double) sequence of strictly positive continuous weights on $\mathbb{D}$ which satisfies

$$
v_{n+1, k} \leqslant v_{n, k} \leqslant v_{n, k+1}, \quad n, k \in \mathbb{N} .
$$

For each $n$ we put $V_{n}:=\left(v_{n, k}\right)_{k}$, and we define the Fréchet spaces

$$
H V_{n}(\mathbb{D}):=\operatorname{proj}_{k} H v_{n, k}(\mathbb{D}), \quad H\left(V_{n}\right)_{0}(\mathbb{D}):=\operatorname{proj}_{k} H\left(v_{n, k}\right)_{0}(\mathbb{D})
$$

as well as the (LF)-spaces

$$
\mathcal{V} H(\mathbb{D}):=\operatorname{ind}_{n} H V_{n}(\mathbb{D}), \quad \mathcal{V}_{0} H(\mathbb{D}):=\operatorname{ind}_{n} H\left(V_{n}\right)_{0}(\mathbb{D}) .
$$

In order to describe the topology of these (LF)-spaces by means of weighted supseminorms, Bierstedt, Meise and Summers [8] associated with $\mathcal{V}$ the system $\bar{V}$ of all those weights $\bar{v}: \mathbb{D} \rightarrow[0, \infty)$ which are upper semicontinuous and have the property that for each $n$ there are $\alpha_{n}>0$ and $k=k(n)$ such that $\bar{v} \leqslant \alpha_{n} v_{n, k}$ on $\mathbb{D}$. The projective hulls of the weighted inductive limits are defined by

$$
\begin{aligned}
& H \bar{V}(\mathbb{D}):=\left\{f \in H(\mathbb{D}) ;\|f\|_{\bar{v}}:=\sup _{z \in \mathbb{D}} \bar{v}(z)|f(z)|<\infty \text { for each } \bar{v} \in \bar{V}\right\}, \\
& H \bar{V}_{0}(\mathbb{D}):=\{f \in H(\mathbb{D}) ; \bar{v}|f| \text { vanishes at } \infty \text { for each } \bar{v} \in \bar{V}\},
\end{aligned}
$$

both endowed with the Hausdorff locally convex topology which is given by the system $\left\{\|\cdot\|_{\bar{v}} ; \bar{v} \in \bar{V}\right\}$ of seminorms. The projective hulls are complete locally convex spaces, and $\mathcal{V} H(\mathbb{D})$ and $\mathcal{V}_{0} H(\mathbb{D})$ are contained in their projective hulls $H \bar{V}(\mathbb{D})$ and $H \bar{V}_{0}(\mathbb{D})$, respectively, with continuous inclusions.

Below we will also make use of the corresponding spaces $C V_{n}(\mathbb{D}), C\left(V_{n}\right)_{0}(\mathbb{D}), \mathcal{V} C(\mathbb{D})$, $\mathcal{V}_{0} C(\mathbb{D}), C \bar{V}(\mathbb{D}), C \bar{V}_{0}(\mathbb{D})$ of continuous functions, defined exactly as above, only replacing $H(\mathbb{D})$ by the space $C(\mathbb{D})$ of all continuous complex-valued functions on $\mathbb{D}$. Moreover, in one result in $\S 4$, weighted (LB)-spaces of holomorphic functions on general open sets $G \subset \mathbb{C}^{N}, N \geqslant 1$, will be considered, but we have preferred to state the definitions here only for the case $G=\mathbb{D}$, which is treated in the rest of the article.

The problem of projective description for spaces of holomorphic functions on $\mathbb{D}$ is to determine when

(1) $\mathcal{V} H(\mathbb{D})=H \bar{V}(\mathbb{D})$ or $V_{0} H(\mathbb{D})=H \bar{V}_{0}(\mathbb{D})$ holds algebraically; and

(2) the space $\mathcal{V} H(\mathbb{D})$ or $\mathcal{V}_{0} H(\mathbb{D})$ is a topological subspace of its projective hull $H \bar{V}(\mathbb{D})$ or $H \bar{V}_{0}(\mathbb{D})$, respectively.

A positive answer to question $(2)$, i.e. when $\mathcal{V} H(\mathbb{D})$ or $\mathcal{V}_{0} H(\mathbb{D})$ is a topological subspace of its projective hull, is of particular importance because it permits us to describe the topology of the weighted (LF)-space of holomorphic functions by means of the weighted sup-seminorms $\|\cdot\|_{\bar{v}}, \bar{v} \in \bar{V}$. 
In case $v_{n, k}=v_{n, k+1}=: v_{n}$ holds for each $n, k$, the space $\mathcal{V} H(\mathbb{D})$ is in fact an (LB)space. As a consequence of the main result of Bierstedt, Meise and Summers $[8,1.6]$, in this case the projective description holds algebraically and topologically in particular if the sequence $\mathcal{V}=\left(v_{n}\right)_{n}$ satisfies the following condition: (S) for each $n$ there is $m>n$ such that $v_{m} / v_{n}$ vanishes at infinity on $\mathbb{D}$. Positive results for $(\mathrm{LB})$-spaces of holomorphic functions on the disc can also be found in $[\mathbf{2 2}]$. There condition $(\mathrm{S})$ is not needed, but all the weights must be normal. In general, the problem of projective description for weighted inductive limits of Banach spaces of holomorphic functions has a negative answer (cf. [12-14]).

The case of (LF)-spaces of holomorphic functions is more complicated. Ehrenpreis [16, pp. 557, 558] showed that the space of real analytic functions $A\left(\mathbb{R}^{N}\right)$ on $\mathbb{R}^{N}$ is not analytically uniform. This implies that the topology of the weighted (LF)-space of entire functions which is isomorphic to the strong dual of the space of real analytic functions cannot be described by means of the canonical weighted sup-seminorms (see also $[\mathbf{2 , 1 7}]$ ). Bonet and Meise [11] recently showed that the topological projective description also fails for the natural weighted inductive limits of spaces of entire functions which arise as the Fourier-Laplace transforms of spaces of ultradistributions of compact support in the non-quasianalytic case. We note that this shows that our Theorem 3.1 ceases to hold for non-radial weights on the complex plane. We refer to the recent survey article by Bierstedt $[\mathbf{3}]$ for more details, motivations and open problems.

Our first main result asserts that if the weights $v_{n, k}$ are chosen in a class $\mathcal{W}$ as described above, then $\mathcal{V}_{0} H(\mathbb{D})$ is a topological subspace of its projective hull. Moreover, the same method of proof shows that in the (LB)-case, whenever the weights $v_{n}$ of the decreasing sequence $\mathcal{V}=\left(v_{n}\right)_{n}$ are all chosen from $\mathcal{W}$, then $\mathcal{V} H(\mathbb{D})$ is a topological subspace of the corresponding weighted inductive limit $\mathcal{V} C(\mathbb{D})=\operatorname{ind}_{n} C v_{n}(\mathbb{D})$ of spaces of continuous functions. Hence by the main result of $[\mathbf{7}]$, we obtain $\mathcal{V} H(\mathbb{D})=H \bar{V}(\mathbb{D})$ algebraically and topologically when the sequence $\mathcal{V}$ satisfies condition (D) of [7]. This last result should be compared with [22, Theorem 3.7], where condition (D) is required in a slightly different form.

Our notation concerning inductive limits, (LB)- and (LF)-spaces, etc., is standard. We refer to $[\mathbf{1 8}, \mathbf{1 9}, \mathbf{2 3}, \mathbf{2 4}]$. For our notation concerning complex analysis see [25].

\section{Results for (LF)-spaces and o-growth conditions}

We start directly with the main theorem of this section.

Theorem 3.1. If $\mathcal{V}=\left(v_{n, k}\right)_{n, k}$ is a sequence of weights in the class $\mathcal{W}$, then $\mathcal{V}_{0} H(\mathbb{D})$ is a topological subspace of its projective hull $H \bar{V}_{0}(\mathbb{D})$.

Proof. By [9, Theorem 1.5], the polynomials are contained and dense in each step $H\left(V_{n}\right)_{0}(\mathbb{D})$, hence also in the inductive limit $\mathcal{V}_{0} H(\mathbb{D})$. According to [8, Lemma 1.2], the conclusion follows if we prove that $\mathcal{V}_{0} H(\mathbb{D})$ and $H \bar{V}_{0}(\mathbb{D})$ induce the same topology on the space $P$ of all polynomials. 
To see this we fix an absolutely convex 0-neighbourhood $U$ in $\mathcal{V}_{0} H(\mathbb{D})$. For each $n$ we choose $k(n) \in \mathbb{N}$ and $\varepsilon_{n}>0$ such that

$$
U_{n}:=\left\{f \in H\left(V_{n}\right)_{0}(\mathbb{D}) ;\|f\|_{v_{n, k(n)}} \leqslant \varepsilon_{n}\right\} \subset 2^{-n} U
$$

For each $n$ let us denote by $D_{n} \geqslant 1$ the constant $D\left(v_{n, k(n)}\right)$ of condition (P2), and let $\lambda_{n}:=C D_{n}\left(\varepsilon_{n}\right)^{-1}$, where $C$ is the constant in condition (P1). For each $m$ we define $w_{m}:=\min \left(\lambda_{1} v_{1, k(1)}, \ldots, \lambda_{m} v_{m, k(m)}\right)$. According to our assumptions on the class $\mathcal{W}$, we have $w_{m} \in \mathcal{W}$ for every $m$; moreover, the sequence $\left(w_{m}\right)_{m}$ is decreasing.

We now need the corresponding spaces of continuous functions. We set

$$
W_{m}:=\left\{g \in C\left(V_{m}\right)_{0}(\mathbb{D}) ;\|g\|_{w_{m}} \leqslant 1\right\}
$$

Clearly, $W_{m} \subset W_{m+1}$ for each $m$, and $W_{m}$ is a 0-neighbourhood in $C\left(V_{m}\right)_{0}(\mathbb{D})$ since

$$
\left\{g \in C\left(V_{m}\right)_{0}(\mathbb{D}) ; \sup _{z \in \mathbb{D}} v_{m, k(m)}(z)|f(z)|<\left(\lambda_{m}\right)^{-1}\right\} \subset W_{m} .
$$

Therefore, $W:=\bigcup_{m} W_{m}$ is an absolutely convex 0-neighbourhood in the weighted inductive limit $\mathcal{V}_{0} C(\mathbb{D})=\operatorname{ind}_{m} C\left(V_{m}\right)_{0}(\mathbb{D})$ of spaces of continuous functions. By [8, Theorem 1.3], there is $\bar{v} \in \bar{V}$ such that

$$
\left\{g \in \mathcal{V}_{0} C(\mathbb{D}) ; \sup _{z \in \mathbb{D}} \bar{v}(z)|g(z)| \leqslant 1\right\} \subset W
$$

Put

$$
W_{0}:=\left\{g \in H \bar{V}_{0}(\mathbb{D}) ; \sup _{z \in \mathbb{D}} \bar{v}(z)|g(z)| \leqslant 1\right\},
$$

which is a 0-neighbourhood in the projective hull. Hence it remains to be seen that every polynomial $q \in\left(c^{2}+1\right)^{-1} W_{0}$ belongs to $U$, where $c \geqslant 1$ is the constant defined before condition (P1). Putting $p:=\left(c^{2}+1\right) q$, we obtain $p \in P \cap W_{0}$, and we have to show that $p \in\left(c^{2}+1\right) U$. Since $p \in W_{0} \cap \mathcal{V}_{0} C(\mathbb{D}) \subset W$, there is $m$ such that $w_{m}|p| \leqslant 1$ on $\mathbb{D}$.

We have

$$
p=\sum_{n=0}^{\infty}\left(R_{n+1}-R_{n}\right) p=R_{1} p+\sum_{n=1}^{\infty}\left(R_{n+1}-R_{n}\right) p,
$$

and the sum is in fact finite. We first treat the term $R_{1} p$.

By the condition before (P1) and the estimate on $w_{m}|p|$, we get

$$
w_{m}\left(r_{1}\right) \sup _{|z|=r_{1}}\left|R_{1} p(z)\right| \leqslant c w_{m}\left(r_{1}\right) \sup _{|z|=r_{1}}|p(z)| \leqslant c .
$$

Choose $s \in\{1, \ldots, m\}$ with $w_{m}\left(r_{1}\right)=\lambda_{s} v_{s, k(s)}\left(r_{1}\right)$. From the second inequality in (P1), applied to the polynomial $R_{1} p$ and $v_{s, k(s)}$, and once more the condition before (P1), we 
conclude

$$
\begin{aligned}
\sup _{z \in \mathbb{D}} v_{s, k(s)}(z)\left|R_{1} p(z)\right| & \leqslant C \sup _{n} v_{s, k(s)}\left(r_{n}\right)\left(\sup _{|z|=r_{n}}\left|\left(R_{n+1}-R_{n}\right) R_{1} p(z)\right|\right) \\
& =C v_{s, k(s)}\left(r_{1}\right) \sup _{|z|=r_{1}}\left|\left(R_{2}-R_{1}\right) R_{1} p(z)\right| \\
& =C\left(\lambda_{s}\right)^{-1} w_{m}\left(r_{1}\right) \sup _{|z|=r_{1}}\left|\left(R_{2}-R_{1}\right) R_{1} p(z)\right| \\
& \leqslant 2 c C\left(\lambda_{s}\right)^{-1} w_{m}\left(r_{1}\right) \sup _{|z|=r_{1}}\left|R_{1} p(z)\right| \\
& \leqslant 2 c^{2} C\left(\lambda_{s}\right)^{-1} \leqslant 2 c^{2} C D_{s}\left(\lambda_{s}\right)^{-1}=2 c^{2} \varepsilon_{s} .
\end{aligned}
$$

This implies $R_{1} p \in 2 c^{2} U_{s} \subset c^{2} U$.

To treat the other term $p-R_{1} p=\sum_{n=1}^{\infty}\left(R_{n+1}-R_{n}\right) p$, we first apply the first inequality in (P1) for $w_{m}$ and the estimate for $w_{m}|p|$ to get

$$
w_{m}\left(r_{n}\right)\left(\sup _{|z|=r_{n}}\left|\left(R_{n+2}-R_{n-1}\right) p(z)\right|\right) \leqslant C
$$

for each $n \in \mathbb{N}$.

Inductively, we can write $\mathbb{N}$ as a disjoint union $\bigcup_{s=1}^{m} J_{s}$ such that

$$
w_{m}\left(r_{j}\right)=\lambda_{s} v_{s, k(s)}\left(r_{j}\right) \quad \text { for } j \in J_{s} \text {. }
$$

Now put, for $s=1, \ldots, m, g_{s}:=\sum_{j \in J_{s}}\left(R_{j+1}-R_{j}\right) p$, which is a polynomial; clearly $p-R_{1} p=\sum_{s=1}^{m} g_{s}$.

We fix $s=1, \ldots, m$ and let $p_{n}^{s}:=\left(R_{n+2}-R_{n-1}\right) p$ if $n \in J_{s}$ and $p_{n}^{s}:=0$ otherwise. The properties of the sequence $\left(R_{n}\right)_{n}$ imply

$$
g_{s}=\sum_{n \in J_{s}}\left(R_{n+1}-R_{n}\right)\left(R_{n+2}-R_{n-1}\right) p=\sum_{n=1}^{\infty}\left(R_{n+1}-R_{n}\right) p_{n}^{s},
$$

and all the sums are finite; hence

$$
\sup _{z \in \mathbb{D}} v_{s, k(s)}(z)\left|g_{s}(z)\right|=\sup _{z \in \mathbb{D}} v_{s, k(s)}(z)\left|\sum_{n=1}^{\infty}\left(R_{n+1}-R_{n}\right) p_{n}^{s}\right| .
$$

Since only a finite number of the $p_{n}^{s}$ are non-zero and all the weights belong to the class $\mathcal{W}$, we can apply $(\mathrm{P} 2)$ and the estimate $(*)$ to conclude

$$
\begin{aligned}
\sup _{z \in \mathbb{D}} v_{s, k(s)}(z)\left|g_{s}(z)\right| & \leqslant D_{s} \sup _{n}\left(\sup _{|z|=r_{n}}\left|p_{n}^{s}(z)\right|\right) v_{s, k(s)}\left(r_{n}\right) \\
& \leqslant D_{s} \sup _{n \in J_{s}}\left(\sup _{|z|=r_{n}}\left|p_{n}^{s}(z)\right|\right) v_{s, k(s)}\left(r_{n}\right) \\
& =D_{s} \sup _{n \in J_{s}}\left(\sup _{|z|=r_{n}}\left|\left(R_{n+2}-R_{n-1}\right) p(z)\right|\right) v_{s, k(s)}\left(r_{n}\right) \\
& \leqslant D_{s}\left(\lambda_{s}\right)^{-1} \sup _{n \in J_{s}}\left(\sup _{|z|=r_{n}}\left|\left(R_{n+2}-R_{n-1}\right) p(z)\right|\right) w_{m}\left(r_{n}\right) \\
& \leqslant D_{s}\left(\lambda_{s}\right)^{-1} C=\varepsilon_{s} .
\end{aligned}
$$


This yields $g_{s} \in U_{s} \subset 2^{-s} U$. Thus

$$
p-R_{1} p=\sum_{s=1}^{m} g_{s} \in \sum_{s=1}^{m} 2^{-s} U \subset U,
$$

hence $p \in\left(c^{2}+1\right) U$, as desired.

The conditions $(w Q)$ and $(Q)$ which appear in the following corollaries were introduced by Vogt $[\mathbf{2 7}]$. Note that (wQ) is always satisfied in the (LB)-case while condition (Q) in this case exactly corresponds to $\mathcal{V}$ regularly decreasing in the sense of [8]. We remark that in [4, Definitions 2.1 and 3.1] the conditions (wQ) and (Q) are explicitly formulated in terms of the sequence $\mathcal{V}$. It follows directly from [4, Proposition 2.4] that under condition (wQ) on the sequence $\mathcal{V}$, every bounded subset of $H \bar{V}(\mathbb{D})$ is contained and bounded in $H V_{n}(\mathbb{D})$ for some $n \in \mathbb{N}$, whence the inductive limit $\mathcal{V} H(\mathbb{D})=\operatorname{ind}_{n} H V_{n}(\mathbb{D})$ is regular. As a direct consequence of [4, Theorem 3.3], under condition (Q) on the sequence $\mathcal{V}$ every bounded subset of $H \bar{V}_{0}(\mathbb{D})$ is contained and bounded in $H\left(V_{n}\right)_{0}(\mathbb{D})$ for some $n \in \mathbb{N}$, whence in this case $\mathcal{V}_{0} H(\mathbb{D})=\operatorname{ind}_{n} H\left(V_{n}\right)_{0}(\mathbb{D})$ is also regular.

Corollary 3.2. If $\mathcal{V}=\left(v_{n, k}\right)_{n, k}$ is a sequence of weights in the class $\mathcal{W}$ which satisfies condition $(Q)$, then

$$
\mathcal{V}_{0} H(\mathbb{D})=H \bar{V}_{0}(\mathbb{D})
$$

algebraically and topologically; in particular, in this case $\mathcal{V}_{0} H(\mathbb{D})$ is complete.

Proof. Corollary 3.2 is an easy consequence of Theorem 3.1 and [4, Theorem 3.3].

Corollary 3.3. If $\mathcal{V}=\left(v_{n, k}\right)_{n, k}$ is a sequence of weights in the class $\mathcal{W}$ which satisfies condition $(w Q)$ and if $\mathcal{V} H(\mathbb{D})$ has a basis of 0-neighbourhoods which are closed for the compact open topology, then we also have

$$
\mathcal{V} H(\mathbb{D})=H \bar{V}(\mathbb{D})
$$

algebraically and topologically; in particular, in this case $\mathcal{V} H(\mathbb{D})$ is also complete.

Proof. By [4, Proposition 2.4], condition (wQ) implies the algebraic equality

$$
\mathcal{V} H(\mathbb{D})=H \bar{V}(\mathbb{D})
$$

The condition on the closed neighbourhoods yields by [5, Proposition 5] that $\mathcal{V} H(\mathbb{D})$ is a topological subspace of $H \bar{V}(\mathbb{D})$ whenever $\mathcal{V}_{0} H(\mathbb{D})$ is a topological subspace of $H \bar{V}_{0}(\mathbb{D})$. At this point it only remains to invoke Theorem 3.1 to finish the proof.

According to [5, Corollary 2 and its proof], the condition on the closed neighbourhoods in Corollary 3.3 is satisfied in the (LB)-case if the sequence $\mathcal{V}=\left(v_{n}\right)_{n}$ is regularly decreasing in the sense of $[\mathbf{8}]$. But in the next section we will prove a better result for the (LB)-case, replacing the regularly decreasing condition by the weaker condition (D) of $[7]$. 


\section{Results for (LB)-spaces and O-growth conditions}

We start this section directly with our main result for weighted (LB)-spaces of holomorphic functions with O-growth conditions.

Theorem 4.1. Let $\mathcal{V}=\left(v_{n}\right)_{n}$ be a decreasing sequence of weights $v_{n}$ in the class $\mathcal{W}$. Then $\mathcal{V} H(\mathbb{D})$ is always a topological subspace of the corresponding weighted inductive limit $\mathcal{V} C(\mathbb{D})$ of spaces of continuous functions.

Proof. We modify the method of proof of Theorem 3.1 in the following way. Let $U$ denote an arbitrary absolutely convex 0-neighbourhood in the (LB)-space $\mathcal{V} H(\mathbb{D})$. For each $n \in \mathbb{N}$ there is $\varepsilon_{n}>0$ such that

$$
U_{n}:=\left\{f \in H v_{n}(\mathbb{D}) ;\|f\|_{v_{n}} \leqslant \varepsilon_{n}\right\} \subset 2^{-n} U .
$$

For each $n$ denote by $D_{n} \geqslant 1$ the constant $D\left(v_{n}\right)$ of condition (P2), and with the constant $C \geqslant 1$ of condition (P1) let again $\lambda_{n}:=C D_{n}\left(\varepsilon_{n}\right)^{-1}, n \in \mathbb{N}$. We define $w_{m}:=$ $\min \left(\lambda_{1} v_{1}, \ldots, \lambda_{m} v_{m}\right), m=1,2, \ldots$, and note that then $w_{m} \in \mathcal{W}$ for each $m$ and that the sequence $\left(w_{m}\right)_{m}$ is decreasing.

We again pass to the corresponding spaces of continuous functions and put

$$
W_{m}:=\left\{g \in C v_{m}(\mathbb{D}) ;\|g\|_{w_{m}} \leqslant 1\right\}, \quad m \in \mathbb{N} .
$$

Then $W:=\bigcup_{m} W_{m}$ is an absolutely convex 0-neighbourhood in $\mathcal{V} C(\mathbb{D})$; let us denote $W_{0}:=W \cap \mathcal{V} H(\mathbb{D})$. We will prove that $\left(2 c^{2}+1\right)^{-1} W_{0} \subset U$, where $c \geqslant 1$ is the constant defined before condition (P1). Fix $F \in\left(2 c^{2}+1\right)^{-1} W_{0}$. Then $f:=\left(2 c^{2}+1\right) F$ belongs to $W_{0}$, hence there is $m \in \mathbb{N}$ such that $w_{m}|f| \leqslant 1$ on $\mathbb{D}$. It remains to show that $f \in\left(2 c^{2}+1\right) U$.

Using the Cesàro means of (the partial sums of) the Taylor series of $f$ about 0 as in $\left[\mathbf{9}\right.$, Proposition 1.2 and its proof], one obtains a sequence $\left(p_{j}\right)_{j}$ of polynomials with

$$
w_{m}\left|p_{j}\right| \leqslant w_{m}|f| \leqslant 1 \quad \text { for each } j \in \mathbb{N}
$$

and $p_{j} \rightarrow f$ in $(H(\mathbb{D})$, co). From this point on we repeat the arguments in the proof of Theorem 3.1 to obtain that

$$
p_{j}=R_{1} p_{j}+\sum_{n=1}^{\infty}\left(R_{n+1}-R_{n}\right) p_{j}
$$

where $R_{1} p_{j} \in 2 c^{2} U_{s(0)}$ for some $s(0) \in\{1, \ldots, m\}$ and

$$
\sum_{n=1}^{\infty}\left(R_{n+1}-R_{n}\right) p_{j} \in \sum_{s=1}^{m} U_{s} .
$$

It follows that each $p_{j}$ belongs to $\left(2 c^{2}+1\right) \sum_{s=1}^{m} U_{s}$. By Montel's Theorem each such $U_{s}$ is compact in $\left(H(\mathbb{D})\right.$, co), and since $p_{j} \rightarrow f$ in the compact-open topology, we can conclude that $f \in\left(2 c^{2}+1\right) \sum_{s=1}^{m} U_{s}$. Finally, we obtain from $U_{s} \subset 2^{-s} U$ that we have $f \in\left(2 c^{2}+1\right)\left(\sum_{s=1}^{m} 2^{-s}\right) U \subset\left(2 c^{2}+1\right) U$, as desired. 
We recall that a sequence $\mathcal{V}=\left(v_{n}\right)_{n}$ of weights $v_{n}$ on $\mathbb{D}$ satisfies condition $(D)$ of $[7]$ if there exists an increasing sequence $\mathcal{X}=\left(X_{m}\right)_{m}$ of subsets of $\mathbb{D}$ so that the following two conditions $(\mathrm{N}, \mathcal{X})$ and $(\mathrm{M}, \mathcal{X})$ are satisfied.

$(\mathbf{N}, \mathcal{X}) \forall m \exists n_{m} \geqslant m: \inf _{X_{m}} \frac{v_{k}}{v_{n_{m}}}>0 \forall k>n_{m}$.

$(\mathbf{M}, \mathcal{X}) \forall n \forall Y \subset \mathbb{D}$ with $Y \cap\left(\mathbb{D} \backslash X_{m}\right) \neq \emptyset$ for all $m \exists n^{\prime}=n^{\prime}(n, Y): \inf _{Y} \frac{v_{n^{\prime}}}{v_{n}}=0$.

Corollary 4.2. Let $\mathcal{V}=\left(v_{n}\right)_{n}$ be a decreasing sequence of weights $v_{n}$ in the class $\mathcal{W}$ which satisfies condition $(D)$. Then we have the algebraic and topological identity

$$
\mathcal{V} H(\mathbb{D})=H \bar{V}(\mathbb{D})
$$

Proof. This corollary is an immediate consequence of Theorem 4.1 and of the fact that condition $(\mathrm{D})$ implies the (algebraic and) topological identity $\mathcal{V} C(\mathbb{D})=C \bar{V}(\mathbb{D})$ by $[\mathbf{7}$, Theorem 6.9$]$.

Theorem 3.7 of $[\mathbf{2 2}]$ is very similar to our Corollary 4.2 , but our proof is more direct and seems a bit more natural.

By Bastin [1] , condition (D) is equivalent to the following condition:

$$
\forall\left(\lambda_{j}\right)_{j}, \lambda_{j}>0, \exists \bar{v} \in \bar{V} \forall n \in \mathbb{N} \forall M>0 \exists m>n: \min \left(\frac{M}{v_{n}}, \frac{1}{\bar{v}}\right) \leqslant \sum_{j=1}^{m} \frac{\lambda_{j}}{v_{j}} .
$$

A similar condition, in which the associated weights of [10] are used, turns out to be necessary for projective description. This is the only place in the present article in which domains $G$ different from $\mathbb{D}$ occur and in which the weights are not required to satisfy conditions (L1), (L2).

Proposition 4.3. Let $G$ denote an open subset of $\mathbb{C}^{N}, N \geqslant 1$, and $\mathcal{V}=\left(v_{n}\right)_{n}$ a decreasing sequence of strictly positive continuous functions on $G$. Then the topological identity $\mathcal{V} H(G)=H \bar{V}(G)$ implies

$$
\forall\left(\lambda_{j}\right)_{j}, \lambda_{j}>0, \exists \bar{v} \in \bar{V} \forall n \in \mathbb{N} \forall M>0 \exists m>n:\left(\min \left(\frac{M}{v_{n}}, \frac{1}{\bar{v}}\right)\right)^{\sim} \leqslant \sum_{j=1}^{m} \frac{\lambda_{j}}{v_{j}}, \quad(+)
$$

where for a strictly positive continuous function $w$ on $G$ the associated function $\tilde{w}$ is defined by

$$
\tilde{w}(z)=\sup \{|f(z)| ; f \in H(G),|f| \leqslant w \text { on } G\}, \quad z \in G .
$$

Proof. Given a sequence $\left(\lambda_{j}\right)_{j}$ of positive numbers, we put

$$
W:=\bigcup_{m \in \mathbb{N}} \sum_{j=1}^{m}\left\{g \in H v_{j}(G) ;\|g\|_{v_{j}} \leqslant \lambda_{j}\right\}
$$


which defines a 0-neighbourhood in $\mathcal{V} H(G)$. By assumption there is $\bar{v} \in \bar{V}$ such that $\left\{f \in \mathcal{V} H(G) ;\|f\|_{\bar{v}} \leqslant 1\right\} \subset W$. Now fix $n \in \mathbb{N}, M>0$ and $f \in H(G)$ with

$$
|f| \leqslant \min \left(\frac{M}{v_{n}}, \frac{1}{\bar{v}}\right)
$$

We have $v_{n}|f| \leqslant M$, hence $f \in H v_{n}(G) \subset \mathcal{V} H(G)$, and $\bar{v}|f| \leqslant 1$, hence $\|f\|_{\bar{v}} \leqslant 1$. From this we conclude $f \in W$; thus, there is $m \in \mathbb{N}$ with

$$
f \in \sum_{j=1}^{m}\left\{g \in H v_{j}(G) ;\|g\|_{v_{j}} \leqslant \lambda_{j}\right\}
$$

whereby $f=\sum_{j=1}^{m} g_{j}$ with $\left|g_{j}\right| \leqslant \lambda_{j} / v_{j}$ on $G$. This finally yields

$$
|f| \leqslant \sum_{j=1}^{m} \frac{\lambda_{j}}{v_{j}}
$$

from which the conclusion follows.

In the case in which $\mathcal{V}=\left(v_{n}\right)_{n}$ is a decreasing sequence of weights $v_{n}$ on $\mathbb{D}$ all of which belong to the class $\mathcal{W}$, the converse of Proposition 4.3 is also true.

Theorem 4.4. Let $\mathcal{V}=\left(v_{n}\right)_{n}$ be a decreasing sequence of weights $v_{n}$ in the class $\mathcal{W}$. Then the topological equality $\mathcal{V} H(\mathbb{D})=H \bar{V}(\mathbb{D})$ holds if and only if

$$
\forall\left(\lambda_{j}\right)_{j}, \lambda_{j}>0, \exists \bar{v} \in \bar{V} \forall n \in \mathbb{N} \forall M>0 \exists m>n:\left(\min \left(\frac{M}{v_{n}}, \frac{1}{\bar{v}}\right)\right)^{\sim} \leqslant \sum_{j=1}^{m} \frac{\lambda_{j}}{v_{j}} .
$$

Proof. From Proposition 4.3 we know that the condition $(+)$ is necessary. To show the sufficiency, we follow the method of proof of Theorem 4.1. Let $U$ denote an arbitrary absolutely convex 0-neighbourhood in $\mathcal{V} H(\mathbb{D})$ and choose for each $n \in \mathbb{N}$ a number $\varepsilon_{n}>0$ with

$$
U_{n}:=\left\{f \in H v_{n}(\mathbb{D}) ;\|f\|_{v_{n}} \leqslant \varepsilon_{n}\right\} \subset 2^{-n} U .
$$

Again, for each $n$ denote by $D_{n} \geqslant 1$ the constant $D\left(v_{n}\right)$ of condition (P2), and with the constant $C \geqslant 1$ of condition (P1) put $\gamma_{j}:=2^{-j}\left(C D_{j}\right)^{-1} \varepsilon_{j}=2^{-j}\left(\lambda_{j}\right)^{-1}, j \in \mathbb{N}$, where $\lambda_{j}$ is as in the proof of Theorem 4.1. Applying the condition with the sequence $\left(\gamma_{j}\right)_{j}$ (instead of $\left.\left(\lambda_{j}\right)_{j}\right)$, there is $\bar{v} \in \bar{V}$ such that $(+)$ holds. We want to prove that with the constant $c>0$ defined before condition (P1) the following inclusion is true:

$$
\left(2 c^{2}+1\right)^{-1}\left\{g \in \mathcal{V} H(G) ;\|g\|_{\bar{v}} \leqslant 1\right\} \subset U .
$$

Then the topologies of $\mathcal{V} H(\mathbb{D})$ and $H \bar{V}(\mathbb{D})$ coincide.

Fix $F$ in the set on the left-hand side of the desired inclusion. Then $f:=\left(2 c^{2}+1\right) F$ is a holomorphic function on $\mathbb{D}$ which, as an element of $\mathcal{V} H(\mathbb{D})$, satisfies

$$
|f| \leqslant \min \left(\frac{M}{v_{n}}, \frac{1}{\bar{v}}\right)
$$


for some $M>0$ and some $n \in \mathbb{N}$. As a consequence of condition $(+)$, there is $m \in \mathbb{N}$ such that

$$
|f| \leqslant \sum_{j=1}^{m} \frac{\gamma_{j}}{v_{j}}=\sum_{j=1}^{m} 2^{-j} \frac{\left(C D_{j}\right)^{-1} \varepsilon_{j}}{v_{j}}
$$

holds. The sum on the right-hand side of the last inequality is less than or equal to

$$
\left(\sum_{j=1}^{m} 2^{-j}\right) \max _{j=1, \ldots, m}\left(\frac{\left(C D_{j}\right)^{-1} \varepsilon_{j}}{v_{j}}\right) \leqslant \max _{j=1, \ldots, m}\left(\frac{\left(C D_{j}\right)^{-1} \varepsilon_{j}}{v_{j}}\right) .
$$

Hence we obtain

$$
\min \left(\lambda_{1} v_{1}, \lambda_{2} v_{2}, \ldots, \lambda_{m} v_{m}\right)|f|=\min _{j=1, \ldots, m}\left(C D_{j}\left(\varepsilon_{j}\right)^{-1} v_{j}\right)|f| \leqslant 1 .
$$

As in the proof of Theorem 4.1, it now follows that $f \in\left(2 c^{2}+1\right) \sum_{s=1}^{m} U_{s}$, so that we get $F \in \sum_{s=1}^{m} U_{s} \subset U$, as desired.

\section{Main example}

Let $\varepsilon_{0}>0$ and $k(0) \in \mathbb{N}$ be given. We denote by $\mathcal{W}=\mathcal{W}\left(\varepsilon_{0}, k(0)\right)$ the set of all the strictly positive continuous radial weights $v$ on $\mathbb{D}$ which satisfy $\lim _{r \rightarrow 1-} v(r)=0$, which are non-increasing on $[0,1)$, and such that the following conditions hold:

$$
\begin{aligned}
& \text { (L1) } \quad \inf _{k} \frac{v\left(r_{k+1}\right)}{v\left(r_{k}\right)} \geqslant \varepsilon_{0}, \\
& \text { (L2) } \limsup _{k \rightarrow \infty} \frac{v\left(r_{k+k(0)}\right)}{v\left(r_{k}\right)}<1-\varepsilon_{0} .
\end{aligned}
$$

The class $\mathcal{W}$ is stable under multiplication by positive scalars and under the formation of finite minima, which follows, for example, from the equivalence of (L1), (L2) with the conditions (U), (L) of Shields and Williams (cf. the end of $\S 2.1$ ).

As in $\left[\mathbf{2 1}\right.$, p. 310] the operator $R_{n}, n \in \mathbb{N}$, is defined for a holomorphic function $f$ on $\mathbb{D}, f(z)=\sum_{k=0}^{\infty} a_{k} z^{k}$, as the convolution with de la Vallée Poussin kernel, i.e.

$$
R_{n} f(z):=\sum_{k=0}^{2^{n}} a_{k} z^{k}+\sum_{k=2^{n}+1}^{2^{n+1}} \frac{2^{n+1}-k}{2^{n}} a_{k} z^{k}
$$

In fact, $R_{n}$ is nothing but the arithmetic mean of the partial sums of index $2^{n}, \ldots, 2^{n+1}-1$ of the Taylor series of $f$. It is easy to see $(\mathrm{cf}$. $[\mathbf{2 0 - 2 2}])$ that $R_{n}: H(\mathbb{D}) \rightarrow H(\mathbb{D})$ is a linear operator which is continuous for the compact open topology and that its range is a finite-dimensional subspace of the polynomials; moreover, $R_{n} R_{m}=R_{\min (n, m)}$ holds for each $n, m$ with $n \neq m$, and for each polynomial $p$ there is $n$ such that $R_{n} p=p$. For the fact that the sequence $\left(R_{n}\right)_{n}$ is uniformly bounded on $H v_{0}(\mathbb{D})$ for each $v \in \mathcal{W}$, see $[\mathbf{2 0}, 2.1]$. 
By $[\mathbf{2 2}, 3.2]$, for each $r \in(0,1)$ and each $f \in H(\mathbb{D})$, we have

$$
\sup _{|z|=r}\left|R_{n} f(z)\right| \leqslant 3 \sup _{|z|=r}|f(z)| .
$$

This implies, for each $v \in \mathcal{W}$ and each polynomial $p$,

$$
\sup _{n}\left(\sup _{|z|=r_{n}}\left|\left(R_{n+2}-R_{n-1}\right) p(z)\right|\right) v\left(r_{n}\right) \leqslant 6 \sup _{n} \sup _{|z|=r_{n}}|p(z)| v\left(r_{n}\right) \leqslant 6\|p\|_{v},
$$

from which the first inequality in condition (P1) follows. The second inequality is formula (3.12) in [22, Lemma 3.3].

To check the condition (P2), we fix $v \in \mathcal{W}$ and a sequence $\left(p_{t}\right)_{t}$ of polynomials of which only finitely many are non-zero. We adapt the arguments in the proofs of $[\mathbf{2 1}, 3.8]$ and $[\mathbf{2 2}, 3.5]$.

We have to estimate $\sup _{z \in \mathbb{D}} v(z)\left|\sum_{t=1}^{\infty}\left(R_{t+1}-R_{t}\right) p_{t}(z)\right|$. Since all the terms are polynomials, the sum is finite and $v \in \mathcal{W}$, we can apply the second inequality in (P1) (i.e. formula (3.12) in [22, Lemma 3.3]) to conclude

$$
\sup _{z \in \mathbb{D}} v(z)\left|\sum_{t=1}^{\infty}\left(R_{t+1}-R_{t}\right) p_{t}(z)\right| \leqslant C \sup _{n} \sup _{|z|=r_{n}}\left|\sum_{t=1}^{\infty}\left(R_{n+1}-R_{n}\right)\left(R_{t+1}-R_{t}\right) p_{t}(z)\right| v\left(r_{n}\right)
$$

with a constant $C>0$ which depends on $v$. As in formula (3.40) in [22], one has

$$
\left(R_{n+1}-R_{n}\right)\left(R_{t+1}-R_{t}\right)= \begin{cases}\left(R_{n}-R_{n-1}\right)\left(R_{n+1}-R_{n}\right) & \text { if } t=n-1 \\ \left(R_{n+1}-R_{n}\right)^{2} & \text { if } t=n, \\ \left(R_{n+2}-R_{n+1}\right) R_{n+1} & \text { if } t=n+1 \\ 0 & \text { for any other } t\end{cases}
$$

Therefore, the last term above can be estimated (redefining only for this formula $p_{0}:=0$ ) by

$$
\begin{aligned}
C \sup _{n}\left\|\left(R_{n}-R_{n-1}\right)\left(R_{n+1}-R_{n}\right) p_{n-1}\right\|_{v}+C \sup _{n}\left\|\left(R_{n+1}-R_{n}\right)^{2} p_{n}\right\|_{v} \\
+C \sup _{n}\left\|\left(R_{n+2}-R_{n+1}\right) R_{n+1} p_{n+1}\right\|_{v} .
\end{aligned}
$$

Invoking the second inequality in (P1) once more, the above sum is estimated by

$$
\begin{aligned}
C^{2} \sup _{n} \sup _{|z|=r_{n-1}}\left|\left(R_{n}-R_{n-1}\right)\left(R_{n+1}-R_{n}\right) p_{n-1}(z)\right| v\left(r_{n-1}\right) \\
+C^{2} \sup _{n} \sup _{|z|=r_{n}}\left|\left(R_{n+1}-R_{n}\right)^{2} p_{n}(z)\right| v\left(r_{n}\right) \\
\quad+C^{2} \sup _{n} \sup _{|z|=r_{n+1}}\left|\left(R_{n+2}-R_{n+1}\right) R_{n+1} p_{n+1}(z)\right| v\left(r_{n+1}\right),
\end{aligned}
$$


which by $(* *)$ is less than or equal to

$$
\begin{aligned}
C^{2} c \sup _{n} \sup _{|z|=r_{n-1}}\left|p_{n-1}(z)\right| v\left(r_{n-1}\right)+C^{2} c \sup _{n} \sup _{|z|=r_{n}}\left|p_{n}(z)\right| v\left(r_{n}\right) & \\
& +C^{2} c \sup _{n} \sup _{|z|=r_{n+1}}\left|p_{n+1}(z)\right| v\left(r_{n+1}\right)
\end{aligned}
$$

for a constant $c$ which depends on $v$. This implies condition (P2).

Acknowledgements. The research presented here was completed during J.B.'s stay at K.D.B.'s institution between May and August 2002. We gratefully acknowledge the support of the Alexander von Humboldt Foundation and of the University of Paderborn. The research of J. Bonet was also partly supported by the MCYT DGI, project no. BFM 2001-2670.

\section{References}

1. F. Bastin, Weighted spaces of continuous functions, Bull. Soc. R. Sci. Liège 59 (1990), $1-81$.

2. C. A. Berenstein And M. A. Dostal, Analytically uniform spaces and their applications to convolution equations, Lecture Notes in Mathematics, vol. 256 (Springer, 1972).

3. K. D. Bierstedt, A survey of some results and open problems in weighted inductive limits and projective description for spaces of holomorphic functions, Bull. Soc. R. Sci. Liège 70 (2001), 167-182.

4. K. D. Bierstedt And J. Bonet, Weighted (LF)-spaces of continuous functions, Math. Nachr. 165 (1994), 25-48.

5. K. D. Bierstedt and J. Bonet, Biduality in (LF)-spaces, Rev. R. Acad. Cien. A 95 (2001), 171-180.

6. K. D. Bierstedt And R. Meise, Induktive Limites gewichteter Räume stetiger und holomorpher Funktionen, J. Reine Angew. Math. 282 (1976), 186-220.

7. K. D. Bierstedt And R. Meise, Distinguished echelon spaces and the projective description of weighted inductive limits of type $\mathcal{V}_{d} \mathcal{C}(X)$, in Aspects of mathematics and its applications, vol. 34, pp. 169-226 (North-Holland Mathematical Library, Amsterdam, 1986).

8. K. D. Bierstedt, R. Meise and W. H. Summers, A projective description of weighted inductive limits, Trans. Am. Math. Soc. 272 (1982), 107-160.

9. K. D. Bierstedt, J. Bonet and A. Galbis, Weighted spaces of holomorphic functions on bounded domains, Michigan Math. J. 40 (1993), 271-297.

10. K. D. Bierstedt, J. Bonet and J. Taskinen, Associated weights and spaces of holomorphic functions, Studia Math. 127 (1998), 137-168.

11. J. BONET AND R. Meise, Ultradistributions of Roumieu type and projective descriptions, J. Math. Analysis Applic. 255 (2001), 122-136.

12. J. Bonet AND S. N. Melikhov, Interpolation of entire functions and projective descriptions, J. Math. Analysis Applic. 205 (1997), 454-460.

13. J. BONET AND J. TASKINEN, The subspace problem for weighted inductive limits of spaces of holomorphic functions, Michigan Math. J. 42 (1995), 259-268.

14. J. Bonet And D. Vogt, On the topological description of weighted inductive limits of spaces of holomorphic and harmonic functions, Arch. Math. (Basel) $\mathbf{7 2}$ (1999), 360-366.

15. P. Domański AND M. Lindström, Sets of interpolation and sampling for weighted Banach spaces of holomorphic functions, Ann. Polon. Math., in press.

16. L. Ehrenpreis, Solution of some problem of division, Part IV, Invertible and elliptic operators, Am. J. Math. 82 (1960), 522-588. 
17. L. Ehrenpreis, Fourier analysis in several complex variables, Tracts in Mathematics, vol. 17 (Wiley, 1970).

18. H. JARCHOW, Locally convex spaces, in Mathematische Leitfäden (Teubner, Stuttgart, 1981).

19. G. KӧтнE, Topological vector spaces, in Grundlehren der Mathematischen Wissenschaften, vol. 237 (Springer, 1979).

20. W. Lusky, On the structure of $H v_{0}(\mathbb{D})$ and $h v_{0}(\mathbb{D})$, Math. Nachr. 159 (1992), 279-289.

21. W. Lusky, On weighted spaces of harmonic and holomorphic functions, J. Lond. Math. Soc. 51 (1995), 309-320.

22. P. Mattila, E. Saksman and J. Taskinen, Weighted spaces of harmonic and holomorphic functions: sequence space representations and projective descriptions, Proc. Edinb. Math. Soc. 40 (1997), 41-62.

23. R. MEISE AND D. Vogt, Introduction to functional analysis (Clarendon, Oxford, 1997).

24. P. PÉRez Carreras And J. Bonet, Barrelled locally convex spaces, Mathematics Studies, vol. 131 (North-Holland, Amsterdam, 1987).

25. W. Rudin, Real and complex analysis, 3rd edn (McGraw-Hill, New York, 1991).

26. A. L. Shields And D. L. Williams, Bounded projections and the growth of harmonic conjugates in the disc, Michigan Math. J. 29 (1982), 3-25.

27. D. Vogt, Regularity properties of LF-spaces, in Progress in functional analysis, Mathematics Studies, vol. 170, pp. 57-84 (North-Holland, Amsterdam, 1992). 